\title{
Representation of the LV 3D Phase Dispersion Using Gated Blood Pool Tomography
}

\author{
JC Souplet ${ }^{1}$, C Léger ${ }^{1}$, V Eder ${ }^{2}$ \\ ${ }^{1}$ Laboratoire d'Électronique, Signaux, Images, Université d'Orléans, Orléans, France \\ ${ }^{2}$ Centre Hospitalier Universitaire de Tours, Tours, France
}

\begin{abstract}
The aim of the work presented here is to compare two new $3 D$ methods of cardiac kinetic analysis with the standard $2 D$ radio nuclide angiography (ERNA). The first new method is a geometric method, based on the evolution of the ventricular $3 D$ reconstruction, obtained after segmentation. The second method directly relies on isotopic data, without segmentation process. The results presented in this paper concern the regional representation and the global comparison of the contraction phase of the left ventricle of the heart. Results show that the $3 D$ methods give more precise phase estimation than the reference ERNA method. This can be related to the isolation of volume of interest within $3 D$ data.
\end{abstract}

\section{Introduction}

Multi-site biventricular pacing (BVP) is a promising new therapy for chronic heart failure based on direct left ventricular (LV) electrical stimulation by pacemakers. It is used to obtain an interventricular synchronization and to improve mechanical pumping efficiency. Gated blood pool tomography (GBP) is a reference examination currently used in Nuclear Medicine to evaluate asynchronism. However, this method presents drawbacks, since surrounding structures (auricles, etc.) are included, leading to inaccurate estimations of the LV ejection fraction and phase (systolic instant, that is to say the instant where the $\mathrm{LV}$ volume is minimum). In order to reduce these artefacts, using $3 \mathrm{D}$ methods seems to be very promising.

This paper presents two new 3D methods used to represent phase dispersion of the LV. The first new method, called ACS for Segmental Kinetic Analysis, estimates LV regional volumes as a function of time. It is a geometrical method since it relies on the segmentation of the LV volume, which is divided into regions of interest. Then, the systolic instant is computed within each region of the reconstructed volumes. The second method, called ACI for Contraction Analysis from
Isotopic data, directly uses isotopic data, targeting the structure of interest within 3D data. Phase analysis is then performed from isolated isotopic data.

The rest of the paper is organized as follows. First, the three methods used in the study are presented. Section 3 compares the results of the 3D methods with the gold standard, using real medical data. Finally some concluding remarks are made.

\section{Methods}

\subsection{D radio nuclide angiography (ERNA)}

Equilibrium planar gamma angiography is a standard examination frequently used in nuclear medicine. Acquisition is done by a camera located on the left side of the thorax (incidence of 45 degrees, as shown in Figure 1). Left and right ventricles are distinct. However, the projection plane is difficult to orient perpendicularly to the axis of the heart. Consequently, ventricular cavities may appear superimposed within the images. Moreover, isotopic images of the heart include radioactive body parts (auricles, aorta, etc.) contained in the projections. These features lead to approximations during LV phase determination.

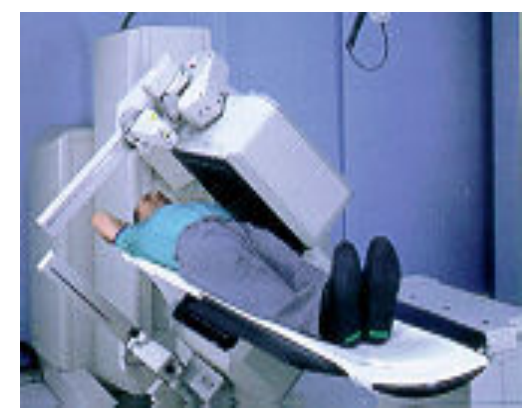

Figure 1. Image acquisition in ERNA

A $2 \mathrm{D}$ record of the $\mathrm{LV}$ is done at 16 instants of the cardiac cycle. Data do not represent the ventricular wall 
itself, but the blood that fills the cavity bounded by this wall. Images represent the radioactivity volume's projection. For each pixel, the global phase of the LV contraction is estimated.

\subsection{Segmental Kinetic Analysis (ACS)}

The ACS method was developed to reconstruct the LV volume [1] as a function of time. Blood pool tomoscintigraphy is used to provide $3 \mathrm{D}$ data, reconstructed by filtered back projection. In such an imaging modality, 3D data are considered as a stack of thin $2 \mathrm{D}$ slices.

The first step of the method consists in segmenting the LV cavity at different instants of the cardiac cycle [2]. An ellipsoidal mask (centred on the LV) is applied on 3D data. Then, data within the mask are thresholded and filtered, in order to get a 2D contour of the cavity in each slice of the stack. By interpolating between all the 2D contours grouped together using Fourier transform, a volume is obtained, as shown in Figure 2. The above stages are performed for all the different instants of the cardiac cycle.

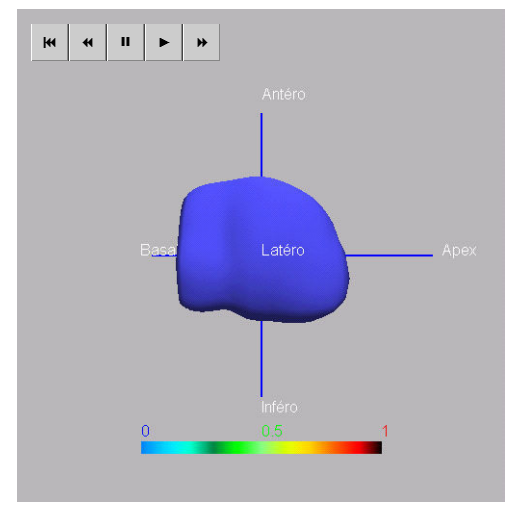

Figure 2. Reconstructed volume of the LV by ACS.

The second step of the method consists in dividing the LV into 17 elementary equal volumes (medical reference), as indicated in Figure 3. The elementary volumes were defined by four adjacent wall points (corresponding to areas that can be mapped onto the different territories of coronary vascularization) and the axis of the whole volume. The variation of elementary volumes during the cardiac cycle was assimilated to the wall motion. It defines 17 volumetric evolution curves, which are used to compute LV regional phases of the contraction. The information of phase is colour encoded and mapped onto a bull's-eye representation.

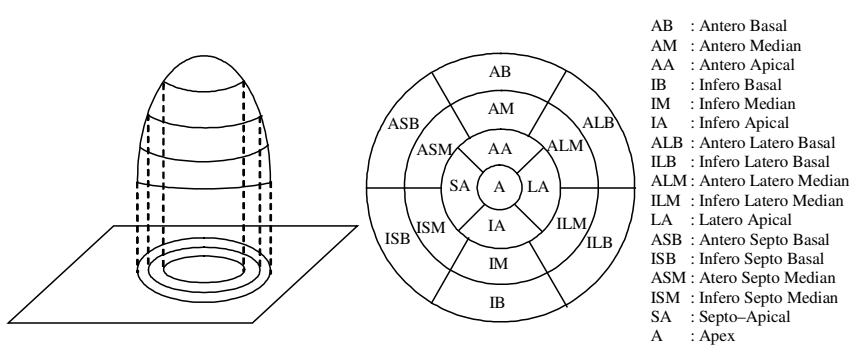

Figure 3. 17 reference segments.

\subsection{Contraction Analysis from Isotopic data (ACI)}

The ACI method was developed to estimate LV contraction parameters using directly raw data. The method is simpler than ACS.

The method can be described in three steps:

1. An ellipsoid mask is applied in each $3 \mathrm{D}$ data of the cardiac cycle, in order to isolate the left ventricle (Figure 4).

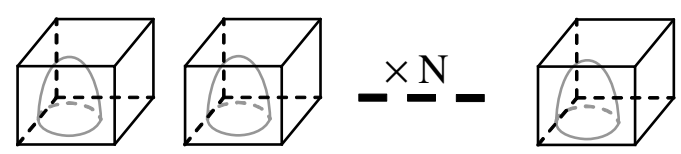

Figure 4. Ventricular volume evolution.

2. 2D images are obtained at each instant of the cardiac cycle by frontal projection of each volume (Figure 5). Applying a mask within 3D data enables peripheral isotopic structures to be removed, thus reducing artefacts in projections, unlike the ERNA method.
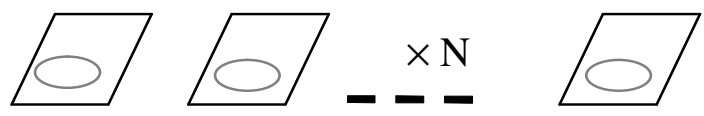

Figure 5. Evolution of the frontal projection of 3D data.

3. Pixel values considered at the same position $(i, j)$ within the projected images are used to build up an evolution curve of the radioactivity in the cardiac cycle. Projecting the volume onto a plane represents the radioactivity rate along the projection axis. It is strongly related to ventricular volume information. Then, each evolution curve represents the volume evolution of the LV along a line parallel to the projection axis, at position $(i, j)$. Figure 6 shows an example of the evolution curve of a projected image pixel. 


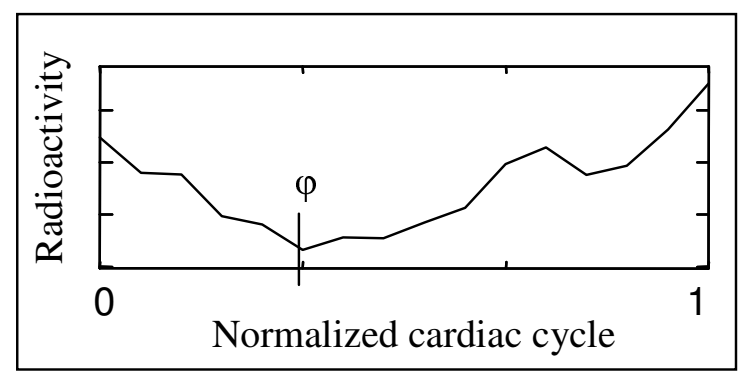

Figure 6. Evolution curve of one pixel after projection of the 3D data inside the elliptic mask.

A phase value is then extracted from each evolution curve. These values are colour encoded and displayed onto a bull's-eye. The images produced are similar to Figure 3, except that colours are not homogeneous within each medical segment, since phase information is provided at pixel level.

\subsection{In vivo study}

Thirty subjects with known cardio myopathies (dilated and ischemic cardio myopathies) were studied. Reference results were obtained after $2 \mathrm{D}$ radio nuclide angiography. SPECT (Single Photon Emission Computed Tomography) gated bloodpool examination provided 3D data, which were transferred to a processing console where ACS and ACI methods were carried out.

\section{Results and discussion}

Figures 7, 8 and 9 show an example of phase dispersion, using the ERNA Standard method, new ACS and new ACI methods, respectively. Bull's eye representation is used to render $3 \mathrm{D}$ information of the phase.

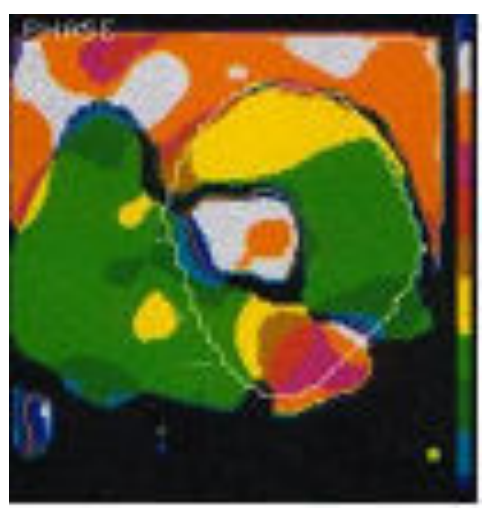

Figure 7. ERNA Results

Qualitatively, the results are similar for all three methods, showing a phase delay in the lower left corner (infero septo basal and infero septo medium sectors). In Figure 8 , colours are uniform within each sector, since the phase is estimated in each sector provided by the ACS method. Conversely, in Figure 9, sector information is superimposed on phase estimation, after processing.

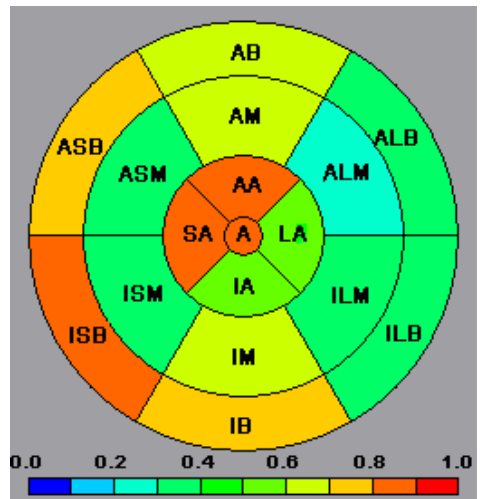

Figure 8. Example of phase information computed with the ACS method.

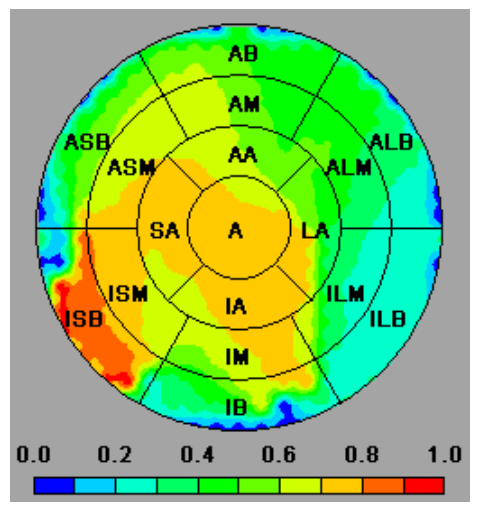

Figure 9. Example of phase information computed with the ACI method.

Figure 10 displays the phase histogram of the same example subject, computed using the ACI method. In this example, the two modes of the histogram indicate a significant asynchronism of the left ventricular contraction.

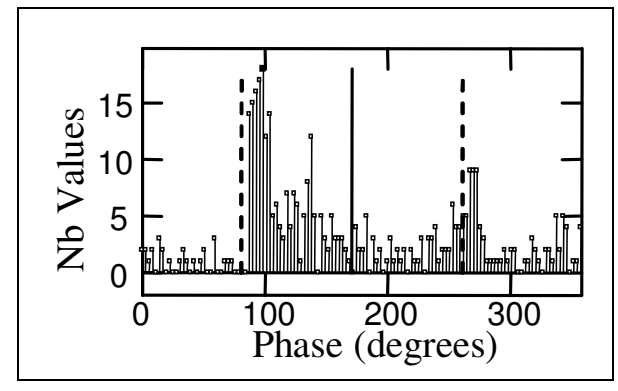

Figure 10. Phase histogram (ACI method). 
Figures 11 and Figure 12 show quantitative results obtained with the population of 30 subjects. Phases obtained with ACS and ACI are respectively compared to the phase given by the reference ERNA method. The slope of the regression line of the ACS phases versus ERNA phases is 1.17. With the ACI Method, the slope of the regression line is 0.98 . Regression lines are very close to the $\mathrm{y}=\mathrm{x}$ line.

The correlation coefficients are 0.73 for ACS and 0.81 for ACI versus ERNA. These coefficients indicate an average correlation between the new methods and the ERNA reference. This is not surprising since the two new methods are highly different from the ERNA methods. According to cardiologists who conducted the experiment, the results provided by the new methods seem to be more realistic than those given by the ERNA method. The difficulty is to prove that the new methods give better results than gold standard ones without relying on reliable reference methods, as is often the case in medical imaging. Nevertheless, making use of true 3D data to remove interference tissues included to $2 \mathrm{D}$ data is certainly a promising way to follow.

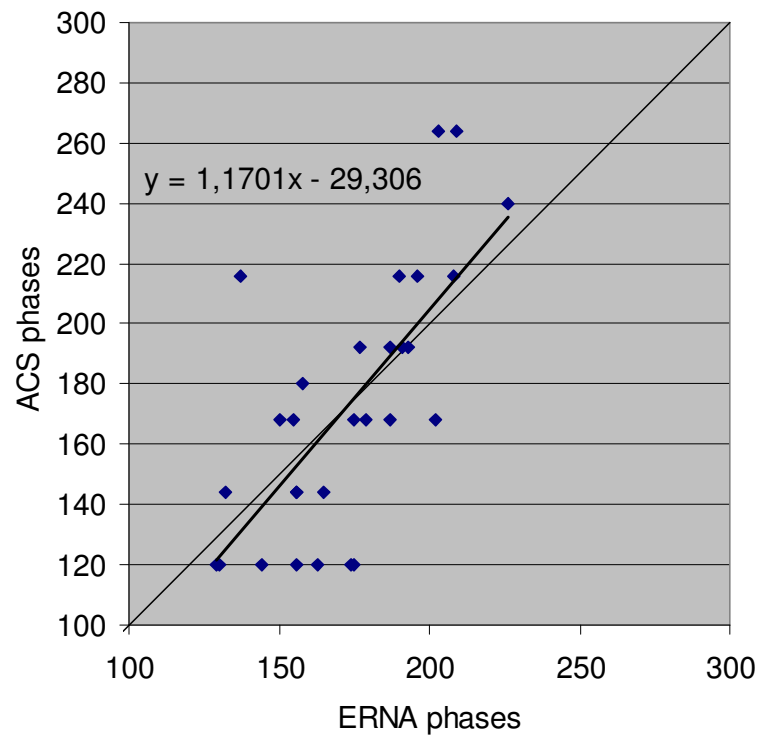

Figure 11. ACS versus ERNA phases.

\section{Conclusion}

This paper presents phase dispersion representations obtained from two new 3D methods, compared to the 2D ERNA reference method. Clinical experiments carried out showed interesting results in left ventricular contraction phase analysis, useful for selecting patients before resynchronization therapy. This preliminary work has to be pursued to better characterize the two new 3D approaches to left ventricle analysis. Then, it will be straightforwardly transposable to the analysis of the right ventricle contraction of the heart.

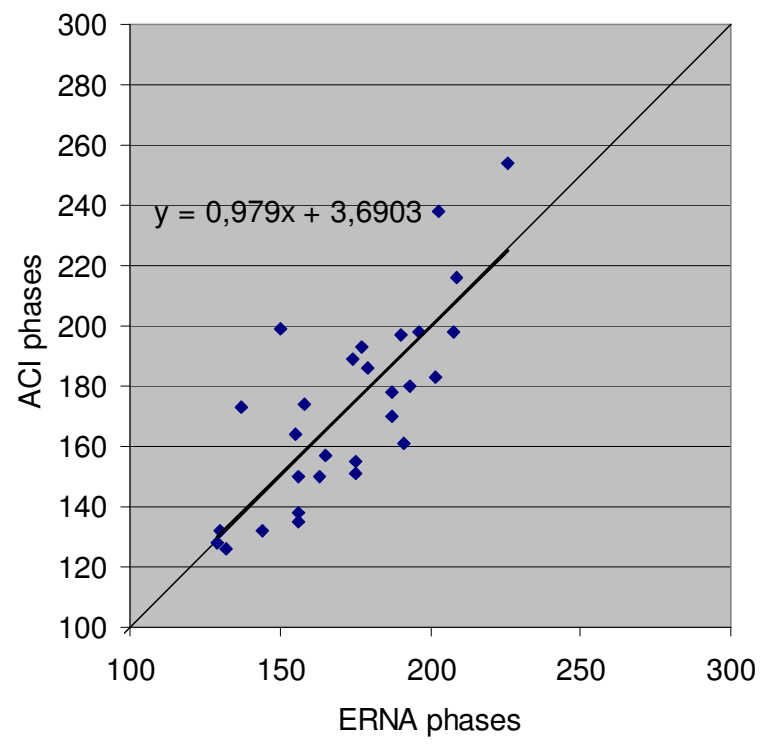

Figure 12. ACI versus ERNA phases

\section{References}

[1] Eder V, Bernis F, Drumm M, Tranquart F, Baulieu F, Pottier JM, Leger C. 3D analysis of left ventricle regional wall motion by using gated bloodpool tomoscintigraphy, Nuclear Medicine Communications, Sept. 2004; 25(9):971-8.

[2] Bonciu $\mathrm{Cl}$, Weber R, Léger C. "4D reconstruction of the left ventricle during a single heart beat, from ultrasound imaging". Image and Vision Computing, Elsevier Eds, April 2001, 19(6): 401-4.

Address for correspondence

Jean-Christophe Souplet

Laboratoire d'Électronique Signaux Images (LESI)

Université d'Orléans

Polytech'Orléans - site Galilée

12 rue de Blois - BP 6744

45067 Orléans cedex 2

France

www.univ-orleans.fr/lesi 